\title{
MicroRNA-101 regulates T-cell acute lymphoblastic leukemia progression and chemotherapeutic sensitivity by targeting Notch1
}

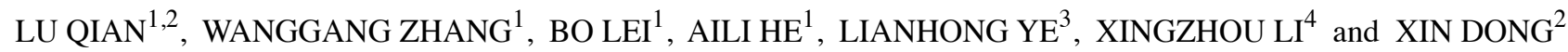 \\ ${ }^{1}$ Department of Hematology, The Second Affiliated Hospital of Xi'an Jiaotong University, Xi'an, Shaanxi 710005; \\ Departments of ${ }^{2}$ Internal Medicine, ${ }^{3}$ Gynaecology and Obstetrics and ${ }^{4}$ Surgery, \\ Xi'an No. 4 Hospital, Xi'an, Shaanxi 710004, P.R. China
}

Received May 11, 2016; Accepted September 12, 2016

DOI: $10.3892 / o r .2016 .5117$

\begin{abstract}
The present study aimed to investigate the role of microRNA (miR)-101 in acute lymphoblastic leukemia progression and chemoresistance. Furthermore, a novel target gene of miR-101 was identified. Here, we confirmed that miR-101 was significantly downregulated in the blood samples of patients with T-cell acute lymphoblastic leukemia (T-ALL) compared with the healthy controls, as determined by reverse transcription quantitative polymerase chain reaction (RTqPCR) analysis. The in vitro experiments demonstrated that miR-101 significantly repressed the proliferation and invasion, and induced potent apoptosis in Jurkat cells, as determined by CCK-8, flow cytometer and cell invasion assays. Luciferase assay confirmed that Notch1 was a target gene of miR-101, and western blotting showed that miR-101 suppressed the expression of Notch1 at the protein level. Moreover, functional restoration assays revealed that Notch1 mediates the effects of miR-101 on Jurkat cell proliferation, apoptosis and invasion. miR-101 enhanced the sensitivity of Jurkat cells to the chemotherapeutic agent adriamycin. Taken together, our results show for the first time that miR-101 acts as a tumor suppressor in T-cell acute lymphoblastic leukaemia and it could enhance chemotherapeutic sensitivity. Furthermore, Notch1 was identified to be a novel target of miR-101. This study indicates that miR-101 may represent a potential therapeutic target for T-cell acute lymphoblastic leukemia intervention.
\end{abstract}

\section{Introduction}

Acute lymphoblastic leukemia (ALL) is an aggressive hematologic malignancy arising from the hematopoietic precursors of lymphoid. It is most common in childhood. T-cell acute

Correspondence to: Professor Wanggang Zhang, Department of Hematology, The Second Affiliated Hospital of Xi'an Jiaotong University, 157 Xiwu Road, Xi'an, Shaanxi 710005, P.R. China E-mail: zhang_wg2003@sina.com

Key words: T-cell acute lymphoblastic leukemia, microRNA-101, Notch1, proliferation, apoptosis, invasion, chemoresistance lymphoblastic leukemia (T-ALL) represents $10-15 \%$ of pediatric and $25 \%$ of adult ALL cases (1), resulting from the malignant transformation of $\mathrm{T}$ cell progenitors. Although the treatment for T-ALL has gradually improved, T-ALL patients with primary resistant or relapsed leukemia have poor prognosis (2-4).

MicroRNAs (miRs) are short non-coding RNAs which negatively regulate protein expression via binding to the complementary sequences within the 3'-untranslated region (UTR) of target mRNA $(5,6)$. Several studies have indicated the importance of microRNAs in the pathogenesis of human leukemia (7-9).

miR-101 is reported as a putative tumor suppressor in several types of cancer, including gastric, prostate cancer, renal cell carcinoma and melanoma (10-13). Recently, increasing studies revealed that miR-101 is also associated with the development of hematological malignancies (14-16). Correia et al (17) have demonstrated that miR-101 is downregulated in T-ALL patient specimens and T-ALL cell lines. However, the exact role of miR-101 in T-ALL progression and chemoresistance remains unclear. Notch1 is a transmembrane receptor that regulates cell growth, differentiation, angiogenesis and metastasis (18-20). Notch1 signaling activation plays key roles in the majority of hematological malignancies including T-ALL $(21,22)$.

In the present study, we detected the expression of miR-101 in the blood samples of patients with T-ALL. The in vitro functional studies were performed on Jurkat cell line to elucidate the effect of miR-101 on cell proliferation, apoptosis, invasion and chemoresistance. Furthermore, whether miR-101 exerts its effect on T-ALL by targeting Notch1 was identified.

\section{Materials and methods}

Clinical samples. The study was approved by the Ethics Committee of The Second Affiliated Hospital of Xi'an Jiaotong University, and all the participants signed a written informed consent for participation in this study. The blood samples were obtained from 25 T-ALL patients and 30 healthy controls.

Cell culture and transfection. The Jurkat and HEK293 cell lines were purchased from the American Type Culture Collection (ATCC; Rockville, MD, USA), and cultured in RPMI-1640 medium (Gibco, Grand Island, NY, USA) supplemented with $10 \%$ fetal bovine serum (FBS; Gibco) at $37^{\circ} \mathrm{C}$ in a 
humidified atmosphere with $5 \% \mathrm{CO}_{2}$. Adriamycin (ADM) was obtained from Sangon Biotech Co., Ltd., (Shanghai, China) and dissolved in phosphate-buffered saline (PBS). Cells were treated with $5 \mu \mathrm{g} / \mathrm{ml}$ adriamycin for $24 \mathrm{~h}$. The cells were transfected with miR-negative control (NC), miR-101 mimic, miR-101 inhibitor, Notch1-pcDNA3.1 or pcDNA3.1 empty vector (Shanghai GenePharma, Co., Ltd., Shanghai, China) using Lipofectamine 2000 (Invitrogen, Waltham, MA, USA) following the manufacturer's protocols. After $6 \mathrm{~h}$, the medium was replaced with fresh medium for further experiments.

Cell proliferation assay. The cells were plated in 96-well plates at $5 \times 10^{3}$ cells/well and allowed to grow for 1-4 days. Subsequently, the cells were incubated with $10 \mu$ l CCK- 8 (Beyotime Institute of Biotechnology, Shanghai, China) at $37^{\circ} \mathrm{C}$ for $4 \mathrm{~h}$. Absorbance was measured at $450 \mathrm{~nm}$ using a microplate reader (DNM-9606; Perlong Medical Equipment Co., Ltd., Beijing, China).

Cell apoptosis assay. Cell apoptosis rate was examined using the Annexin V-FITC and propidium iodide (PI) apoptosis kit (Nanjing KeyGen Biotech, Co., Ltd., Nanjing, China) following the manufacturer's protocols. Briefly, the cells were washed with PBS, and dual-stained with Annexin V and PI. The apoptosis cells were detected by a FACSCalibur flow cytometer (Becton-Dickinson, Sparks, MD, USA).

Cell invasion assay. Invasiveness of Jurkat cells were performed using Transwell inserts ( $5 \mu \mathrm{m}$ pore size; Corning Inc., Corning, NY, USA) coated with Matrigel (BD Biosciences, Bedford, MA, USA). The matrix solutions were loaded into the upper well of Transwell chambers, and incubated at $37^{\circ} \mathrm{C}$ for $1 \mathrm{~h}$. Each group of cells were resuspended in FBS-free medium and placed into the upper Transwell chambers. The lower chambers were filled with RPMI-1640 medium containing $10 \% \mathrm{FBS}$. After incubation at $37^{\circ} \mathrm{C}$ for $24 \mathrm{~h}$, the non-invading cells on the top of the membrane were removed by wiping. The invading cells on the lower face of the membrane were fixed with $3.7 \%$ paraformaldehyde, and stained with crystal violet staining solution (Beyotime Institute of Biotechnology). Cells in the lower compartments were also counted.

Luciferase assay. The wild-type Notch1 3' untranslated region (UTR) carrying a putative miR-101 binding site, and the mutant Notch1 3'UTR were inserted into psiCHECK-2 vector (Promega, Madison, WI, USA). HEK293 cells were co-transfected with miR-NC/miR-101 and wild-type/mutant Notch1-3'UTR using Lipofectamine 2000. The Renilla luciferase reporter vector was transfected as an internal control in each assay. Luciferase activity was measured $24 \mathrm{~h}$ after transfection using the Luciferase Reporter assay system (Promega).

Reverse transcription quantitative polymerase chain reaction (RTqPCR). Total RNA was extracted using the RNeasy/ miR Neasy Mini kit (Qiagen, Limburg, The Netherlands) according to the manufacturer's protocols. Total RNA (5 ng) was used for reverse transcription, using the RevertAid ${ }^{\mathrm{TM}}$ First Strand cDNA Synthesis kit (Fermentas, Vilnius, Lithuania). The primers for miR-101 were the exact sequence of mature miR-101. They were purchased from GenScript (Nanjing,

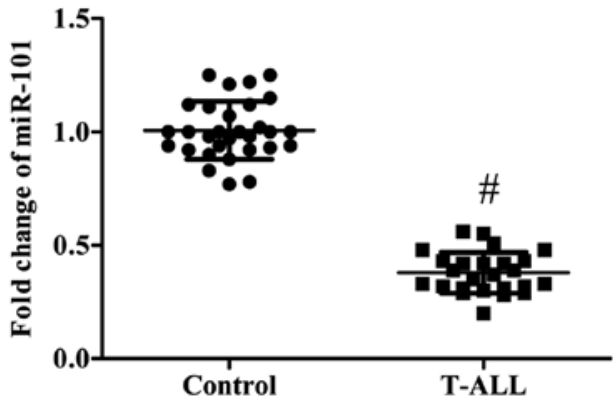

Figure 1. Fold change of miR-101 expression in the blood samples of healthy controls and patients with T-ALL. ${ }^{\text {P }}<0.01$ compared with the control. miR, microRNA; T-ALL, T-cell acute lymphoblastic leukemia.

China). PCR was performed with the SYBR-Green PCR Master Mix (Applied Biosystems, Foster City, CA, USA) on the ABI PRISM 7700 Sequence detection system (Applied Biosystems). The relative expression of miR-101 was calculated by the $2^{-\Delta \Delta \mathrm{Ct}}$ method that was normalized to the U6 internal control.

Western blot analysis. Whole cell lysates were prepared using ice-cold RIPA buffer supplemented with the protease inhibitor (Beyotime Institute of Biotechnology). The protein concentration was determined using the Bradford reagent (Pierce, Rockford, IL, USA). An equal amount of protein $(20 \mu \mathrm{g})$ was resolved by $10 \%$ SDS-PAGE, and then transferred to nitrocellulose membranes (Bio-Rad Laboratories, Inc., Hercules, CA, USA). The membranes were blocked with 5\% non-fat milk at room temperature for $2 \mathrm{~h}$, and then incubated with the specific antibodies at $4{ }^{\circ} \mathrm{C}$ overnight, including rabbit polyclonal to Notch1 (1:600; cat. no. 3881-100; BioVision Incorp., Milpitas, CA, USA) and rabbit polyclonal to $\beta$-actin $(1: 1,000$; cat. no. C1836; Applygen Technologies, Inc., Beijing, China). After washing with TBST, the membranes were further incubated with HRP-labelled goat anti-rabbit $\operatorname{IgG}(1: 1,000$; cat. no. C2226; Applygen Technologies) at $37^{\circ} \mathrm{C}$ for $1 \mathrm{~h}$. The immunoreactive bands were visualized using the chemiluminescent substrate (Pierce).

Statistical analysis. All data represent at least three independent experiments and are expressed as the means \pm SD. Student's t-test or one-way ANOVA test was used to compare the differences between groups. $\mathrm{P}<0.05$ was considered statistically significant.

\section{Results}

Expression of miR-101 in the blood samples of patients with $T$-ALL. RT-qPCR was performed to detect miR-101 expression in the blood samples of 25 T-ALL patients and 30 healthy controls. Compared with the healthy controls, the expression of miR-101 was significantly downregulated in the blood samples of patients with T-ALL ( $\mathrm{P}<0.01$; Fig. 1).

Effect of miR-101 on cell proliferation, apoptosis and invasion of Jurkat cells. The Jurkat cells were transfected with the miR-NC, miR-101 mimic or miR-101 inhibitor, and then RT-qPCR analysis was performed to detect miR-101 

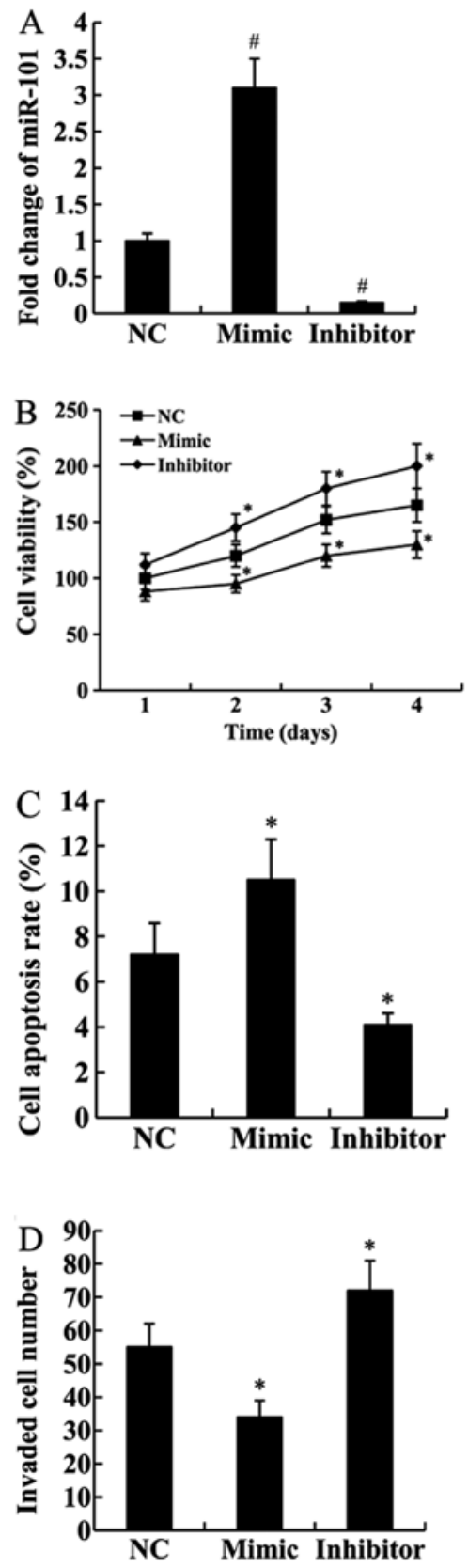

Figure 2. Effect of miR-101 on cell proliferation, apoptosis and invasion of Jurkat cells. (A) Fold change of miR-101 expression in Jurkat cells transfected with the miR-NC, miR-101 mimic or miR-101 inhibitor. (B) Cell proliferation; (C) cell apoptosis; (D) cell invasion of Jurkat cells transfected with the miR-NC, miR-101 mimic or miR-101 inhibitor. ${ }^{*} \mathrm{P}<0.05$ and ${ }^{\#} \mathrm{P}<0.01$ compared with the miR-NC. miR, microRNA; NC, negative control.

expression. The results showed that compared with the miR-NC-transfected cells, the expression of miR-101 was significantly upregulated in miR-101 mimic-transfected cells but downregulated in miR-101 inhibitor-transfected cells ( $\mathrm{P}<0.01$; Fig. 2A).

To elucidate the effect of miR-101 on T-ALL progression, the in vitro functional assays were performed on Jurkat cells. As shown in Fig. 2B, the proliferation ability of Jurkat cells transfected with the miR-101 mimic was significantly weaker than those transfected with the miR-NC $(\mathrm{P}<0.05)$. In addition, the cell proliferation ability was enhanced in miR-101 inhibitor transfected cells compared with the control cells $(\mathrm{P}<0.05)$.

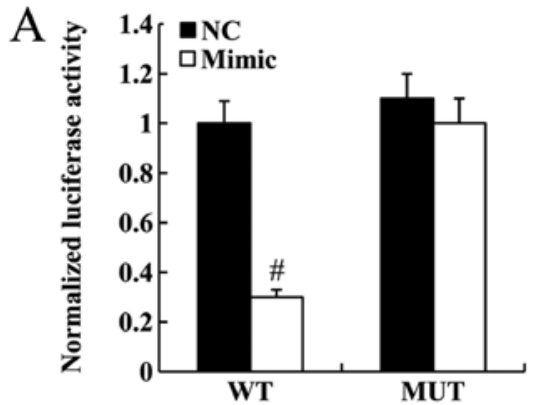

B

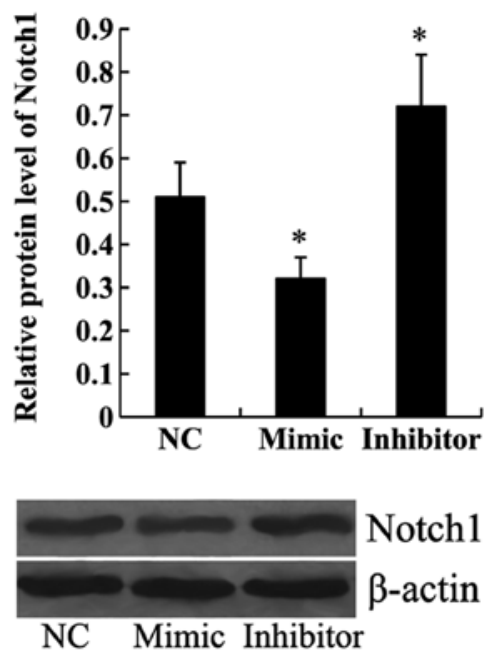

Figure 3. Notch1 is a direct target of miR-101. (A) The transcription activity of wild-type or mutant Notch1-3'UTR in Jurkat cells transfected with the miR-NC or miR-101 mimic. (B) Relative protein level of Notch1 in Jurkat cells transfected with the miR-NC, miR-101 mimic or miR-101 inhibitor. ${ }^{*} \mathrm{P}<0.05$ and ${ }^{\#} \mathrm{P}<0.01$ compared with the miR-NC. miR, microRNA; NC, negative control; WT, wild-type; MUT, mutant; UTR, untranslated region.

We examined whether miR-101 could affect cell apoptosis using FCM analysis. We found that compared with the miR-NC-transfected cells, cell apoptosis rate was significantly increased in the cells transfected with the miR-101 mimic but decreased in the cells transfected with the miR-101 inhibitor (P<0.05; Fig. 2C).

Cell invasion assay confirmed that the invasive ability of Jurkat cells was inhibited by transfection with the miR-101 mimic $(\mathrm{P}<0.05)$. By contrast, transfection with the miR-101 inhibitor significantly increased cell invasive ability $(\mathrm{P}<0.05$; Fig. 2D).

Notch1 is a direct target of miR-101. To determine whether Notch1 was a target gene of miR-101, wild-type or mutant Notch1-3'UTR was transfected into the HEK293 cells along with the miR-NC or miR-101 mimic. Luciferase assay demonstrated that miR-101 mimic significantly inhibited the transcription activity of wild-type Notch1-3'UTR $(\mathrm{P}<0.01)$. However, the transcription activity of mutant Notch1-3'UTR was not affected by the transfection of miR-101 mimic (Fig. 3A). Furthermore, the results from western blot analysis revealed that the expression of Notch1 protein was significantly downregulated in the miR-101 mimic-transfected cells and upregulated in the miR-101 inhibitor-transfected cells $(\mathrm{P}<0.05$; Fig. 3B). 

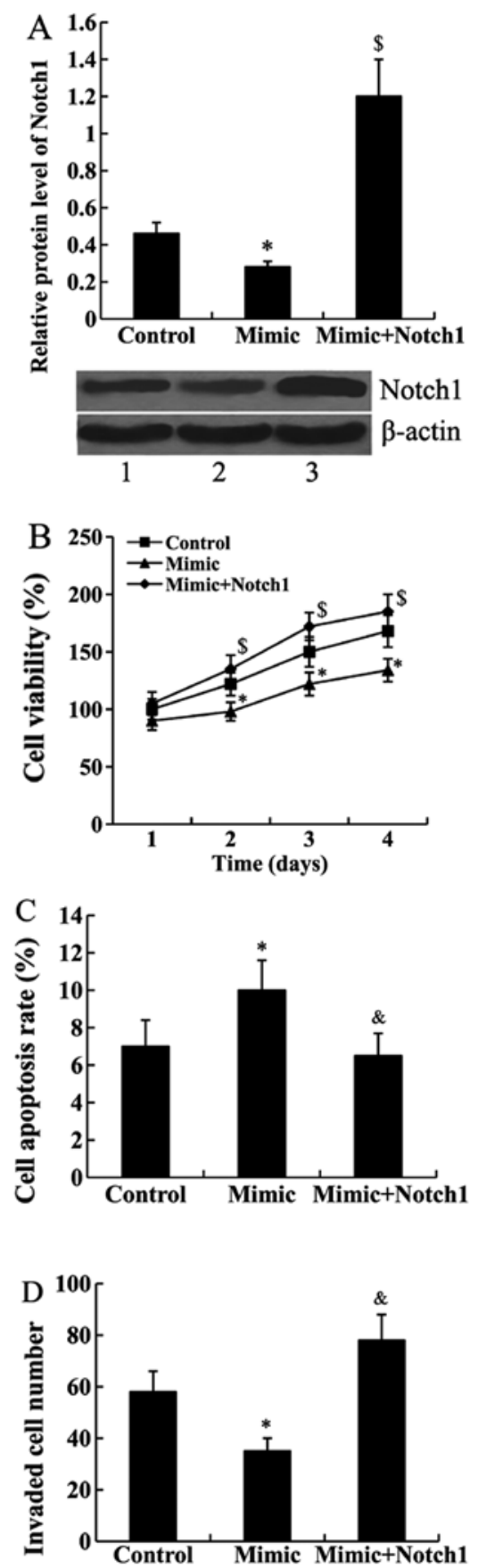

Figure 4. Notch1 attenuated the effect of miR-101 on cell proliferation, apoptosis and invasion. (A) Relative protein level of Notch1 in Jurkat cells transfected with the miR-101 mimic and Notch1-pcDNA3.1. Lane 1, control; lane 2, Mimic; lane 3, Mimic+Notch1. (B) Cell proliferation; (C) cell apoptosis; (D) cell invasion of Jurkat cells transfected with the miR-101 mimic and Notch1-pcDNA3.1. "P $<0.05$ compared with the control; ${ }^{\circ} \mathrm{P}<0.05$ and ${ }^{\$} \mathrm{P}<0.01$ compared with the miR-101 mimic. miR, microRNA; NC, negative control.

Notchl attenuates the effect of miR-101 on cell proliferation, apoptosis and invasion. Notch1-pcDNA3.1 was transfected into the Jurkat cells to overexpress Notch 1. As shown in Fig. 4A, the results from the western blot analysis confirmed that the relative protein level of Notch1 was significantly upregulated in the cells transfected with the miR-101 mimic and Notch1-pcDNA3.1 compared with the cells transected with the miR-101 mimic only $(\mathrm{P}<0.01)$.
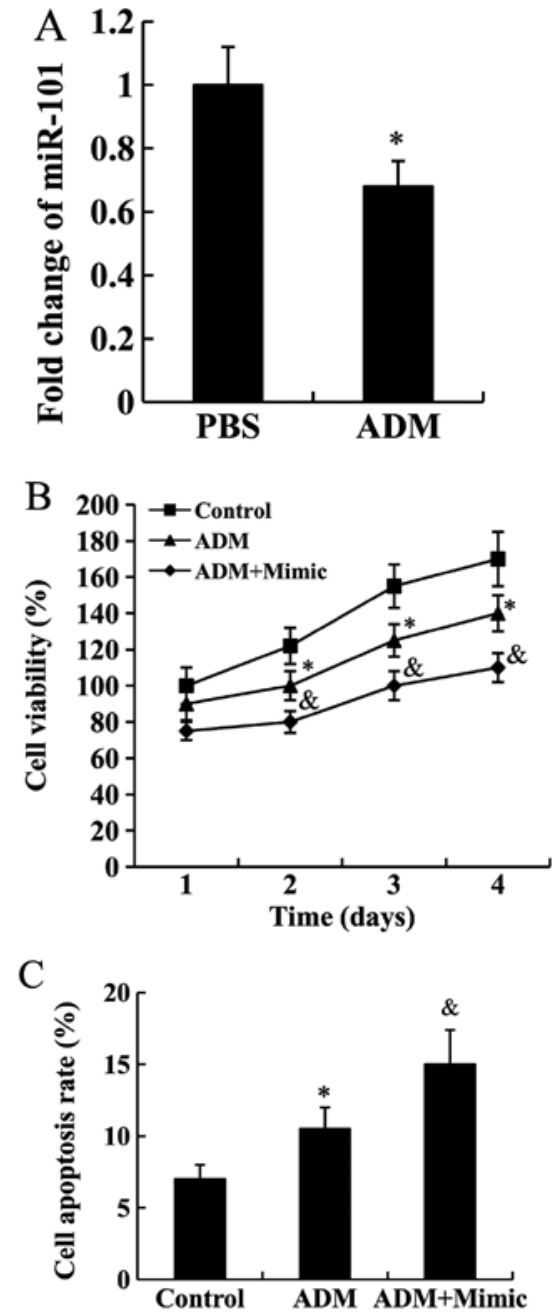

Figure 5. miR-101 enhances drug sensitivity of Jurkat cells. (A) Fold change of miR-101 expression in Jurkat cells treated with adriamycin. ${ }^{*} \mathrm{P}<0.05$ compared with PBS. (B) Cell proliferation; (C) cell apoptosis of Jurkat cells treated with adriamycin and transfected with the miR-101 mimic. ${ }^{*} \mathrm{P}<0.05$ compared with PBS; ${ }^{\circ} \mathrm{P}<0.05$ compared with ADM. miR, microRNA; ADM, adriamycin.

Subsequently, CCK-8, FCM and cell invasion assays were performed to determine whether Notch1 mediates the effect of miR-101 on cell proliferation, apoptosis and invasion. We found that the suppressed cell proliferation and invasion abilities by miR-101 mimic transfection were attenuated by Notch1 overexpression in Jurkat cells. In addition, miR-101 mimic-induced cell apoptosis rate was inhibited by Notch1pcDNA3.1 transfection (Fig. 4B-D).

miR-101 enhanced drug sensitivity of Jurkat cells. Jurkat cells were treated with $5 \mu \mathrm{g} / \mathrm{ml}$ adriamycin for $24 \mathrm{~h}$, and the expression of miR-101 was subsequently detected. We found that miR-101 expression was significantly decreased in Jurkat cells following treatment with adriamycin $(\mathrm{P}<0.05$; Fig. 5A).

Jurkat cells transfected with miR-101 mimic were subjected to adriamycin treatment, then cell proliferation and apoptosis rate were analyzed. As shown in Fig. 5B and C, adriamycin was able to inhibit cell proliferation and promote cell apoptosis $(\mathrm{P}<0.05)$, these effects were enhanced by miR-101 mimic $(\mathrm{P}<0.05)$. 


\section{Discussion}

The expression of miR-101 has been extensively studied in hematological malignancies. It was reported that miR-101 was downregulated in samples of Burkitt lymphoma (14) and adult ALL cases (23). Fallah et al (24) revealed that miR-101 was upregulated in patients with newly diagnosed chronic myeloid leukemia in chronic phase. Recently, Correia et al (17) demonstrated that miR-101 is downregulated in T-ALL patient specimens and T-ALL cell lines, and it may be involved in the development of T-cell acute lymphoblastic leukemia. In the present study, we detected miR-101 expression in the blood samples of patients with T-ALL. We found that compared with the healthy controls, the expression of miR-101 was significantly downregulated in T-ALL patients. This finding was consistent with the previous study on T-ALL patient specimens and T-ALL cell lines (17).

miR-101 has been reported to be downregulated in various types of cancer, and it can repress cell proliferation and metastatic ability in gallbladder, liver, breast and ovarian cancers (25-28). Thus, miR-101 is widely recognized as a tumor suppressor. miR-101 is also downregulated in T-ALL, however, its function in T-ALL has not been reported. We further performed in vitro studies to determine the effect of miR-101 on Jurkat cell proliferation, apoptosis and invasion. Both the gain- and loss-of-function experiments revealed that miR-101 could inhibit cell proliferation and invasion, and increase apoptosis of Jurkat cells, suggesting the tumorsuppressive role of miR-101 in T-ALL. Furthermore, the present study provided the first evidence that miR-101 could enhance the drug sensitivity of Jurkat cells to adriamycin.

Notch1 is a well-known regulator that plays an oncogenic role in many malignancies (29). Notch1 mutation is present in over $50 \%$ of T-cell acute lymphoblastic leukemias (T-ALL) (30). Constitutive activation of Notch1 signaling can induce T-ALL in murine models. Suppression of Notch1 signaling leads to the decreased cell proliferation and increased cell apoptosis in the context of T-ALL $(31,32)$. Furthermore, Notch1 is related to chemoresistance $(33,34)$, a major cause of poor prognosis in T-ALL. Using miRanda (http://www.microrna. org), Notch1 was predicted to be a target of miR-101. In the present study, we confirmed that miR-101 could inhibit the transcription activity of Notch1-3'UTR using luciferase assay. In addition, the expression of Notch1 protein was downregulated by miR-101. These results identified Notch1 as a direct target gene of miR-101. Furthermore, we found that Notch1 overexpression could attenuate the effect of miR-101 on cell proliferation, apoptosis and invasion of Jurkat cells. These findings suggested that miR-101 exerts its effect on T-ALL at least partially by downregulating Notch1.

In conclusion, the present study revealed the tumorsuppressive role of miR-101 in T-ALL by directly targeting Notch1. In addition, miR-101 was able to enhance adriamycin sensitivity in Jurkat cells. miR-101 could be potentially of value in T-ALL therapy.

\section{Acknowledgements}

The present study was supported by the foundation of Xi'an Municipal Bureau of Science and Technology [no. SF1317(2)].

\section{References}

1. Ferrando AA, Neuberg DS, Staunton J, Loh ML, Huard C, Raimondi SC, Behm FG, Pui CH, Downing JR, Gilliland DG, et al: Gene expression signatures define novel oncogenic pathways in $\mathrm{T}$ cell acute lymphoblastic leukemia. Cancer Cell 1: 75-87, 2002.

2. Pui $\mathrm{CH}$, Robison LL and Look AT: Acute lymphoblastic leukaemia. Lancet 371: 1030-1043, 2008.

3. Goldberg JM, Silverman LB, Levy DE, Dalton VK, Gelber RD, Lehmann L, Cohen HJ, Sallan SE and Asselin BL: Childhood T-cell acute lymphoblastic leukemia: The Dana-Farber Cancer Institute acute lymphoblastic leukemia consortium experience. J Clin Oncol 21: 3616-3622, 2003.

4. Oudot C, Auclerc MF, Levy V, Porcher R, Piguet C, Perel Y, Gandemer V, Debre M, Vermylen C, Pautard B, et al: Prognostic factors for leukemic induction failure in children with acute lymphoblastic leukemia and outcome after salvage therapy: The FRALLE 93 study. J Clin Oncol 26: 1496-1503, 2008.

5. Ambros V: The functions of animal microRNAs. Nature 431: 350-355, 2004.

6. Bartel DP: MicroRNAs: Genomics, biogenesis, mechanism, and function. Cell 116: 281-297, 2004.

7. Seca H, Almeida GM, Guimarães JE and Vasconcelos MH: miR signatures and the role of miRs in acute myeloid leukaemia. Eur J Cancer 46: 1520-1527, 2010.

8. Giza DE and Calin GA: microRNA and chronic lymphocytic leukemia. Adv Exp Med Biol 889: 23-40, 2015.

9. Yendamuri S and Calin GA: The role of microRNA in human leukemia: A review. Leukemia 23: 1257-1263, 2009.

10. Zhou X, Xia Y, Li L and Zhang G: MiR-101 inhibits cell growth and tumorigenesis of Helicobacter pylori related gastric cancer by repression of SOCS2. Cancer Biol Ther 16: 160-169, 2015.

11. Hao Y, Gu X, Zhao Y, Greene S, Sha W, Smoot DT, Califano J, Wu TC and Pang X: Enforced expression of miR-101 inhibits prostate cancer cell growth by modulating the COX-2 pathway in vivo. Cancer Prev Res (Phila) 4: 1073-1083, 2011.

12. Sakurai T, Bilim VN, Ugolkov AV, Yuuki K, Tsukigi M, Motoyama T and Tomita Y: The enhancer of zeste homolog 2 (EZH2), a potential therapeutic target, is regulated by miR-101 in renal cancer cells. Biochem Biophys Res Commun 422: 607-614, 2012.

13. Luo C, Merz PR, Chen Y, Dickes E, Pscherer A, Schadendorf D and Eichmüller SB: MiR-101 inhibits melanoma cell invasion and proliferation by targeting MITF and EZH2. Cancer Lett 341: 240-247, 2013.

14. Robertus JL, Kluiver J, Weggemans C, Harms G, Reijmers RM, Swart Y, Kok K, Rosati S, Schuuring E, van Imhoff G, et al: MiRNA profiling in B non-Hodgkin lymphoma: A MYC-related miRNA profile characterizes Burkitt lymphoma. Br J Haematol 149: 896-899, 2010.

15. Cheng J, Guo S, Chen S, Mastriano SJ, Liu C, D'Alessio AC, Hysolli E, Guo Y, Yao H, Megyola CM, et al: An extensive network of TET2-targeting MicroRNAs regulates malignant hematopoiesis. Cell Rep 5: 471-481, 2013.

16. Papakonstantinou N, Ntoufa S, Chartomatsidou E, Papadopoulos G, Hatzigeorgiou A, Anagnostopoulos A, Chlichlia K, Ghia P, Muzio M, Belessi C, et al: Differential microRNA profiles and their functional implications in different immunogenetic subsets of chronic lymphocytic leukemia. Mol Med 19: 115-123, 2013.

17. Correia NC, Melão A, Póvoa V, Sarmento L, Gómez de Cedrón M, Malumbres M, Enguita FJ and Barata JT: microRNAs regulate TAL1 expression in T-cell acute lymphoblastic leukemia. Oncotarget 7: 8268-8281, 2016.

18. Sethi N and Kang Y: Notch signalling in cancer progression and bone metastasis. Br J Cancer 105: 1805-1810, 2011.

19. Roy M, Pear WS and Aster JC: The multifaceted role of Notch in cancer. Curr Opin Genet Dev 17: 52-59, 2007.

20. Sahlgren C, Gustafsson MV, Jin S, Poellinger L and Lendahl U: Notch signaling mediates hypoxia-induced tumor cell migration and invasion. Proc Natl Acad Sci USA 105: 6392-6397, 2008.

21. Liu N, Zhang J and Ji C: The emerging roles of Notch signaling in leukemia and stem cells. Biomark Res 1: 23, 2013.

22. Zou J, Li P, Lu F, Liu N, Dai J, Ye J, Qu X, Sun X, Ma D, Park J, et al: Notch1 is required for hypoxia-induced proliferation, invasion and chemoresistance of T-cell acute lymphoblastic leukemia cells. J Hematol Oncol 6: 3, 2013. 
23. Ninomiya S, Tyybäkinoja A, Borze I, Räty R, SaarinenPihkala UM, Usvasalo A, Elonen E and Knuutila S: Integrated analysis of gene copy number, copy neutral $\mathrm{LOH}$, and microRNA profiles in adult acute lymphoblastic leukemia. Cytogenet Genome Res 136: 246-255, 2012.

24. Fallah P, Amirizadeh N, Poopak B, Toogeh G, Arefian E, Kohram F, Hosseini Rad SM, Kohram M, Teimori Naghadeh H and Soleimani M: Expression pattern of key microRNAs in patients with newly diagnosed chronic myeloid leukemia in chronic phase. Int J Lab Hematol 37: 560-568, 2015.

25. Su H, Yang JR, Xu T, Huang J, Xu L, Yuan Y and Zhuang SM: MicroRNA-101, down-regulated in hepatocellular carcinoma, promotes apoptosis and suppresses tumorigenicity. Cancer Res 69: 1135-1142, 2009

26. Liu XY, Liu ZJ, He H, Zhang C and Wang YL: MicroRNA-101-3p suppresses cell proliferation, invasion and enhances chemotherapeutic sensitivity in salivary gland adenoid cystic carcinoma by targeting Pim-1. Am J Cancer Res 5: 3015-3029, 2015.

27. Li JT, Jia LT, Liu NN, Zhu XS, Liu QQ, Wang XL, Yu F, Liu YL, Yang AG and Gao CF: MiRNA-101 inhibits breast cancer growth and metastasis by targeting CX chemokine receptor 7. Oncotarget 6: 30818-30830, 2015

28. Zheng HB, Zheng XG and Liu BP: miRNA-101 inhibits ovarian cancer cells proliferation and invasion by down-regulating expression of SOCS-2. Int J Clin Exp Med 8: 20263-20270, 2015 .
29. Capaccione KM and Pine SR: The Notch signaling pathway as a mediator of tumor survival. Carcinogenesis 34: 1420-1430, 2013.

30. O'Neil J, Calvo J, McKenna K, Krishnamoorthy V, Aster JC, Bassing CH, Alt FW, Kelliher $\mathrm{M}$ and Look AT: Activating Notch1 mutations in mouse models of T-ALL. Blood 107: 781-785, 2006.

31. Chan SM, Weng AP, Tibshirani R, Aster JC and Utz PJ: Notch signals positively regulate activity of the mTOR pathway in T-cell acute lymphoblastic leukemia. Blood 110: 278-286, 2007.

32. Real PJ, Tosello V, Palomero T, Castillo M, Hernando E, de Stanchina E, Sulis ML, Barnes K, Sawai C, Homminga I, et al: Gamma-secretase inhibitors reverse glucocorticoid resistance in $\mathrm{T}$ cell acute lymphoblastic leukemia. Nat Med 15: 50-58, 2009.

33. Nefedova Y, Cheng P, Alsina M, Dalton WS and Gabrilovich DI: Involvement of Notch-1 signaling in bone marrow stromamediated de novo drug resistance of myeloma and other malignant lymphoid cell lines. Blood 103: 3503-3510, 2004.

34. Nefedova Y, Sullivan DM, Bolick SC, Dalton WS and Gabrilovich DI: Inhibition of Notch signaling induces apoptosis of myeloma cells and enhances sensitivity to chemotherapy. Blood 111: 2220-2229, 2008. 